\title{
[Letter to the Editor]
}

\section{Accelerated RNA-RNA hybridization by concentrated guanidinium thiocyanate solution in single-step RNA isolation}

Single-step RNA isolation by guanidinium thiocyanate (GuSCN)-acid-phenolchloroform extraction is a popular method used in many molecular biology laboratories (1). With this method, successful isolation of purified and undegraded RNA relies on the properties of GuSCN. This compound is a powerful protein denaturant and when used at high concentrations essentially inactivates all cellular RNases. The exact molecular mechanism of its action is unknown. However, it has been shown that the weak hydration of GuSCN ions contributes to the disruption of hydrogen bonds and interference with hydrophobic interactions in proteins (2). Several commercial RNA isolation kits, including TRI Reagent manufactured by Molecular Research Center (Cincinnati, OH), TRlzol by Invitrogen (Carlsbad, CA) or Thermo Fisher Scientific (Waltham, MA), and QIAzol Lysis Reagent by Qiagen (Valencia, CA), contain GuSCN, which guarantees efficient and reproducible isolation of high-quality total RNA from cultured cells and tissues.

Thompson and Gillespie (3) were the first to discover that concentrated GuSCN solutions promote DNA-DNA, DNA-RNA, and RNA-RNA hybridization. In their studies, the catalytic effect of GuSCN was attributed to the reduction of melting temperatures of hybrids as well as the optimum temperature of hybrid formation. Hybridization with 0.5-1.5 kb radioactive riboprobes was observed in GuSCN-solubilized cells, and the rate of hybridization in the presence of GuSCN, measured for DNA-RNA hybrids, was 50-100x greater than that in 50\% formamide solution.

We hypothesized that sense and antisense (or complementary) transcripts, abundant in different cell types (4), could also form RNA-RNA duplexes if cellular RNA was isolated by commercial kits containing concentrated GuSCN solution (Figure 1A). First, we studied the hybridization of synthetic complementary transcripts of 452 and 414 nucleotides, containing a 103 nucleotide complementary region derived from exon 23 of $A B C A 9$ (5) (Supplementary Material). To identify the catalytic component of TRIzol, we tested the following 3 solutions commonly used in single-step RNA isolation (1): $0.2 \mathrm{M}$ sodium acetate, $\mathrm{pH} 4.0$ ( $\mathrm{NaOAc}$ ); acidic phenol equilibrated with $0.2 \mathrm{M} \mathrm{NaOAc}$; and 4 M GuSCN. In 4 experiments (Figure 1B), equimolar amounts of the complementary transcripts were added sequentially to 100 $\mu \mathrm{L}$ of each solution to obtain a final concentration of $10 \mathrm{~nm}$ for each transcript. After each addition, the solutions were carefully mixed and incubated for 5-10 min at room temperature. RNAs from the $\mathrm{NaOAc}$ and GuSCN solutions were precipitated with an equal volume of isopropanol, and those from the phenol phase were first extracted with an equal volume of $0.2 \mathrm{M} \mathrm{NaOAc}$ followed by isopropanol precipitation. RNAs from the TRIzol solution were isolated by the standard protocol (1), consisting of extraction with $20 \mu \mathrm{L}$ chloroform and precipitation with $50 \mu \mathrm{L}$ isopropanol. After washing with $150 \mu \mathrm{L} 70 \%$ ethanol, all RNAs were dissolved in $6 \mu \mathrm{L}$ TE (10 $\mathrm{mM}$ Tris- $\mathrm{HCl}, \mathrm{pH}$ 7.5, $1 \mathrm{mM}$ EDTA). These RNAs were further divided into 2 aliquots, one of which was treated with a mixture of RNase A (10 $\mu \mathrm{g} / \mathrm{mL}$ ) and RNase T1 (25 U/ $\mathrm{mL}$ ) in RNase protection assay (RPA) buffer (10 mM Tris- $\mathrm{HCl}, \mathrm{pH}$ 7.4, 300 mM NaCl, 5 mM EDTA). Finally, both aliquots (-/+ RNase) were directly loaded onto a $1.5 \%$ agarose $1 \times$ TBE gel containing ethidium bromide (Sigma, St. Louis, MO) and singlestranded RNAs (ssRNAs) were separated from double-stranded RNAs (dsRNAs) by electrophoresis. Figure $1 \mathrm{~B}$ shows that the 0.2 M NaOAc solution had no effect (Lanes 3 and 4) and the acidic phenol produced only partial hybridization of 10\% of transcripts (Lanes 5 and 6), as determined from a comparison of Lanes 6 and 11. As expected, RNA-RNA hybridization was catalyzed by $4 \mathrm{M}$ GuSCN solution, and a comparable effect was observed with TRIzol (Lanes 7-11). Therefore, consistent with the previous study of Thompson and Gillespie (3), these results suggest that GuSCN is the primary component of TRIzol causing RNA-RNA hybridization.

To rule out the possibility that the RNase-resistant dsRNA observed in the GuSCN-catalyzed reactions (Figure $1 \mathrm{~B}$ ) is due to the formation of secondary structures in either transcript, we tested the RNase resistance of the complementary transcripts by incubating them: (i) individually; (ii) after being added together to a single volume (100 $\mu \mathrm{L})$ of TRIzol; or (iii) after being added to 2 separate $(50 \mu \mathrm{L}+50 \mu \mathrm{L})$ volumes of TRIzol that were then mixed together. RNAs from these incubations were isolated by the standard protocol (1). As shown in Supplementary Figure S1, no intramolecular RNase-resistant duplexes were observed for either transcript incubated alone (Lanes 1-4), confirming that the formation of dsRNA was intermolecular. This reaction occurred regardless of how the transcripts were mixed (Lanes 5-8). These results suggest that the individual transcripts do not form strong intramolecular secondary structures that could be resistant to RNases and that only hybridization between the complementary transcripts results in dsRNA formation.

We further wanted to determine the lowest concentration of transcripts sufficient for RNA-RNA hybridization catalyzed by GuSCN. Similar to the experiment described above for TRIzol (Figure 1B, Lanes 9 and 10), we determined the RNA-RNA hybridization for the 1-10 nM concentration range of complementary transcripts. Figure 2A shows that within this concentration range, the hybridization reaction is quantitative (Lanes 1-6, compare Lanes 6 and 7) and most likely does not depend on the complexity of RNA because the addition of total RNA does not reduce the level of hybridization (Lanes 8-14). Initially, we hypothesized that addition of competing total RNA could increase non-specific hybridization and thus reduce the extent of specific RNA- 
RNA hybridization; however, this was clearly not the case. Instead, an increase in dsRNA formation in the presence of total RNA was observed, which may be explained by the differences in the RNase to RNA ratio due to the presence or absence of total RNA, thus resulting in different degrees of digestion in the two series of experiments.

We next performed RNA isolation from human cells using TRIzol (1) with the following modifications. Five hybridization reactions at transcript concentrations of $0.1 \mathrm{nM}, 30 \mathrm{pM}, 10 \mathrm{pM}, 3 \mathrm{pM}$, and $1 \mathrm{pM}$ were performed as described for Figure $2 \mathrm{~A}$, except that transcripts were added directly to TRIzol-lysed cells in 6-well plates. Each well contained $300 \mu \mathrm{L}$ TRIzol and $\sim 1 \times 10^{6}$ human teratocarcinoma cells (NTera2D1). To prevent an excessive local concentration of added transcripts, careful manual rotation of the viscous solution was performed immediately after each addition. RNAs were isolated by extraction with chloroform, precipitation with isopropanol, washing with ethanol and dissolving in 6 $\mu \mathrm{L}$ TE, as described above. Purified RNAs were divided into two aliquots, one of which was treated with a mixture of RNases A and $\mathrm{T} 1$ and the other kept as a control. RNase-resistant dsRNAs were detected by an RPA using ${ }^{32}$ P-labeled riboprobe (5). Before loading onto the high-resolution 40-cm-long 6\% polyacrylamide gel, RNA samples were normalized to the lowest concentration to ensure equal loading. After electrophoresis, the gel was vacuum-dried and exposed to a Phosphorlmager screen (Bio-Rad, Hercules, CA). Bands corresponding to protected RNAs were quantitated using Quantity One 1-D Analysis Software (Bio-Rad). The dsRNA/ssRNA ratio was calculated and averaged from two independent experiments (Figure 2B, bar diagram). Nearly quantitative hybridization occurred at a transcript concentration of $0.1 \mathrm{nM}$ (Figure 2B, Lanes 1 and 2). However, at lower concentrations, only some of the transcripts form dsRNA, most likely due to chemical equilibrium (Lanes 3-10). Because of the variation between experiments, we repeated the isolation protocol and averaged the results from two different experiments. Figure 2B (bar diagram) shows that at the lowest concentration used (1 pM), hybridization of $~ 15 \%$ of the transcripts was detected, although the signal appeared close to the limit of detection. However, we believe that by using RT-PCR, a more sensitive method,

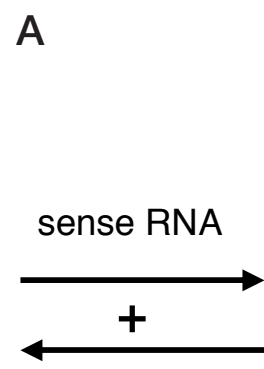

antisense RNA

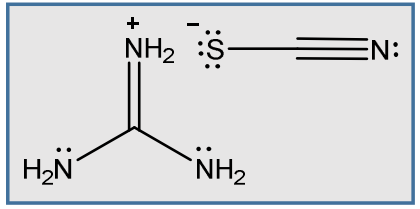

(GuSCN)

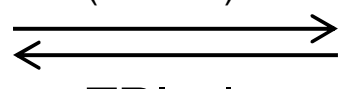

TRIzol

\section{(cell lysate)}
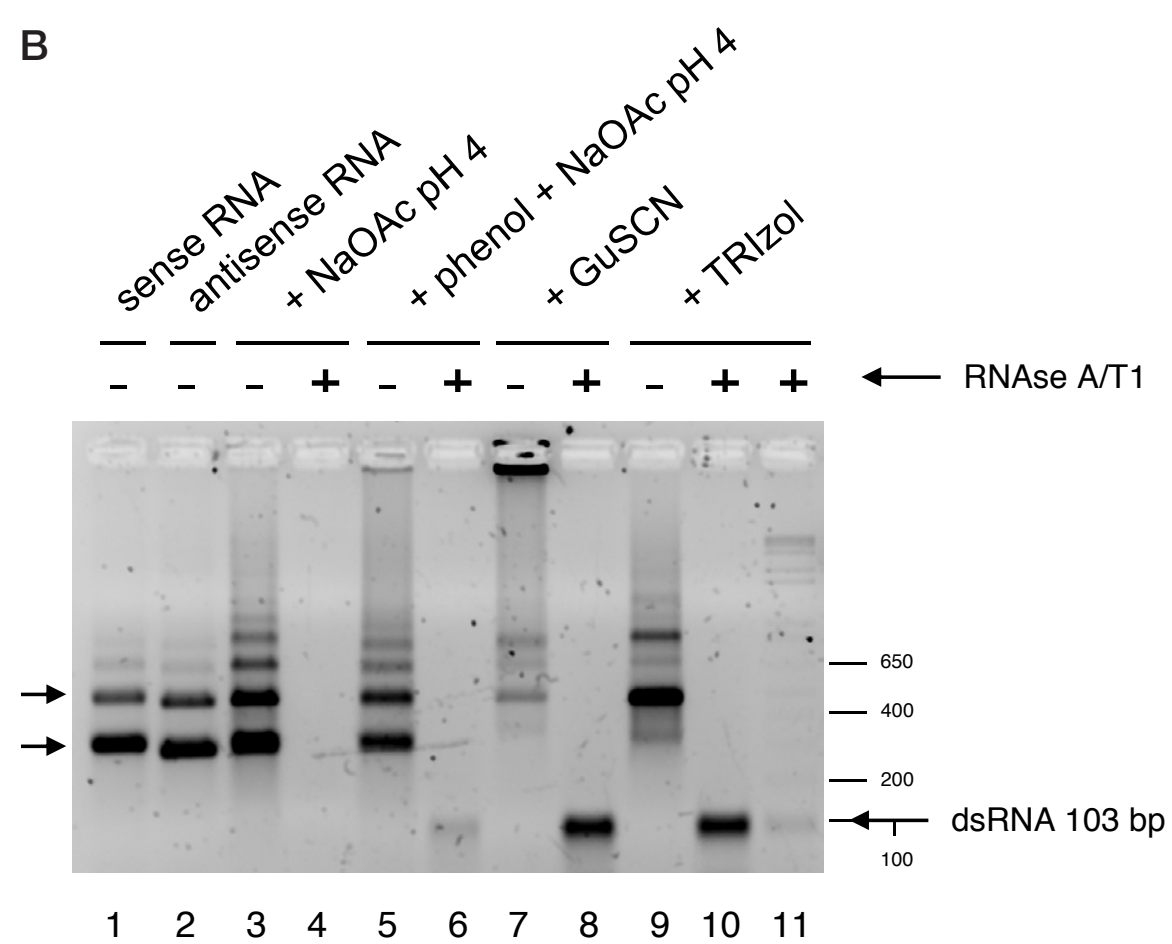

Figure 1. RNA-RNA hybridization catalyzed by guanidinium thiocyanate (GuSCN). (A) Schematic representation of accelerated hybridization of complementary transcripts catalyzed by GuSCN in TRIzol solution during RNA isolation. (B) Hybridization of complementary transcripts in $0.2 \mathrm{M}$ $\mathrm{NaOAc}, \mathrm{pH} 4.0$ (Lanes 3 and 4); acidic phenol, pH 4.0 (Lanes 5 and 6); 4 M GuSCN (Lanes 7 and 8); or TRIzol (Lanes 9-11), as determined by -/+ RNase A/T1 treatment and agarose gel electrophoresis. Lanes 1 and 2: untreated complementary transcripts. Note that single-stranded transcripts were predominantly in monomeric and dimeric forms. Formation of the latter was most likely due to the presence of complementary short (6-8 nucleotide) palindromic sequences in their $5^{\prime}$ ends derived from the multiple cloning site of the vector (see Supplementary Material). Lane 11 contained one-tenth of the sample amount in lane 10 loaded in a background (20 ng) of $1 \mathrm{~kb}$ Plus DNA Ladder (Invitrogen). The origin of the precipitate in the well of Lane 7 is unclear.

much smaller amounts of dsRNA at lower transcript concentrations ( $<1 \mathrm{pM})$ may be detected. Therefore, these experiments demonstrated that complementary transcripts at picomolar concentrations are still able to hybridize and form dsRNA in the concentrated GuSCN solution. In addition, using a modified RPA (hybridization in $4 \mathrm{M}$ GuSCN), we demonstrated that dsRNAs ranging from 90-290 bp can easily be formed and detected at low concentrations (data not shown).
We were curious to see how many publications describe detection of dsRNA and the use of RNA isolation with commercial kits containing GuSCN. A PubMed search using the keywords dsRNA, RNA duplex, and TRIzol yielded at least a dozen publications describing the detection of putative regulatory dsRNAs in vivo (6-17). In all of these publications, a sensitive RT-PCR was used to detect dsRNAs ranging from 100-500 bp. Surprisingly, however, none of them had addressed the potential of 


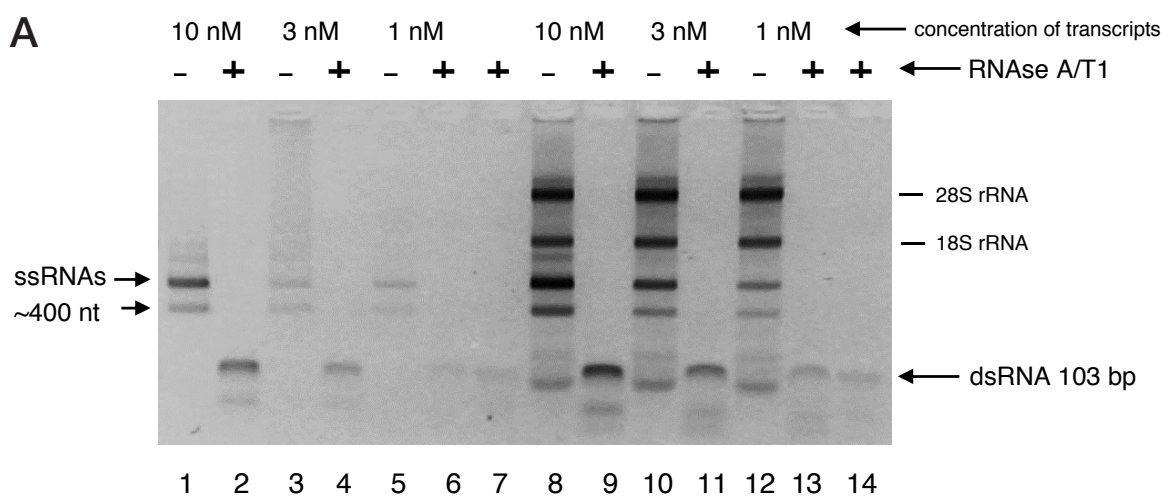

।
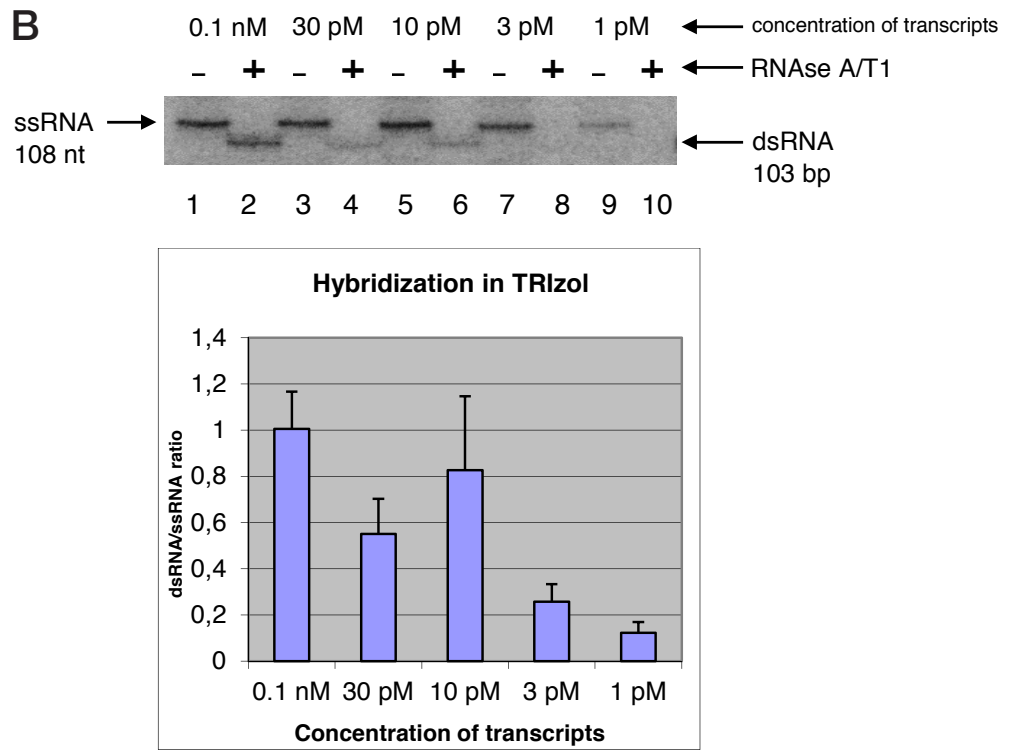

Figure 2. RNA-RNA hybridization of low concentrations of complementary transcripts catalyzed by the guanidinium thiocyanate (GuSCN) in TRIzol. (A) Hybridization of synthetic sense and antisense transcripts at nanomolar concentrations in TRIzol in the absence (Lanes 1-7) and presence of total RNA (1 $\mu \mathrm{g}$ ) (Lanes 8-14), containing 5S, 18S, and 28S ribosomal RNA. The formation of dsRNA was determined by -/+ RNase A/T1 treatment and agarose gel electrophoresis. To reveal quantitative hybridization, one-tenth of the RNA sample amounts loaded in Lanes 1 and 8 were loaded in Lanes 7 and 14, respectively. (B) Hybridization of complementary transcripts at picomolar concentrations (Lanes 1-10) determined by -/+ RNase A/T1 treatment and detection by RNase protection assay (RPA). The bar graph shows the the ratio of dsRNA/ssRNA averaged for two independent experiments.

RNA-RNA hybridization in the concentrated GuSCN solution as demonstrated earlier by Thompson and Gillespie (3).

Finally, we would like to propose an explanation for why dsRNA formation in vivo could be inefficient. Our earlier transient transfection experiments revealed that two transcription units with opposite orientation embedded in a single plasmid or separated into two different plasmids both gave rise to dsRNAs (5). This result was unexpected and may be explained by GuSCN catalysis during RNA isolation. It is reasonable to assume that complementary transcripts derived from cis-acting convergent transcription units are more likely to form an RNA duplex because, spatially, they are generated at the same site of synthesis. In trans, however, plasmids with different transcription units may be separated into different transcription factories that are distant from one another. Alternatively, even transcription units in the same transcription factory may not be sterically close enough to allow hybridization (as they might be in a single vector). Furthermore, it is generally accepted that nascent pre-mRNAs emerging from the RNA polymerase complex are immediately associated with heterogeneous ribonucleoproteins (hnRNPs) (18), therefore reducing their chances of RNA-RNA hybridization. In support of the inhibitory role of hnRNPs, it has been demonstrated that pre-incubation of either of two complementary RNAs with hnRNPs strongly inhibited the nucleation step between complementary strands, whereas pre-incubation of both RNAs with hnRNPs abolished their hybridization (19). Altogether, these results demonstrate that hybridization between complementary transcripts may be prevented by co-transcriptional and sequence-specific binding of hnRNPs. We cannot exclude the possibility that overexpression might cause some of the transcripts to escape hnRNP binding, allowing them to form dsRNA; however, even in this case, the cis orientation may be preferred.

To prove that the observed dsRNA hybrids are real and not artifacts generated by GuSCN-catalyzed hybridization of complementary ssRNAs during RNA isolation, we propose the following three tests: (i) RNA isolation under nondenaturing conditions, as described by Krystal et al. (20); (ii) immunoprecipitation of dsRNA from unfractionated cells by dsRNA-specific antibodies (21); and (iii) inclusion of dsRNAspecific RNases III or V1 in the early stage(s) of RNA isolation in the negative control. We believe that the use of these methods would help verify that potentially novel regulatory dsRNAs isolated from cells are genuine.

\section{Author contributions}

T.M. and M.S. performed the experiments and analyzed the data. M.S. wrote and revised the manuscript. Both authors read and approved the final version.

\section{Acknowledgments}

We thank Richard Tamme and Ly Villo for comments and critical reading of the manuscript. M.S. was supported by grants from the Estonian Science Foundation (ETF8381) and the Estonian Research Council (PUT1221).

\section{Competing interests}

The authors declare no competing interests.

\section{References}

1. Chomczynski, P. and N. Sacchi. 1987. Single-step method of RNA isolation by acid guanidinium thiocyanate-phenol-chloroform extraction. Anal. Biochem. 162:156-159.

2. Mason, P.E., G.W. Neilson, C.E. Dempsey, A.C. Barnes, and J.M. Cruickshank. 2003. The hydration structure of guanidinium and thiocyanate ions: implications for protein stability in aqueous solution. Proc. Natl. Acad. Sci. USA 100:4557-4561. 
3. Thompson, J. and D. Gillespie. 1987. Molecular hybridization with RNA probes in concentrated solutions of guanidine thiocyanate. Anal. Biochem. 163:281-291.

4. Yelin, R., D. Dahary, R. Sorek, E.Y. Levanon, O. Goldstein, A. Shoshan, A. Diber, S. Biton, et al. 2003. Widespread occurrence of antisense transcription in the human genome. Nat. Biotechnol. 21:379-386

5. Kaer, K., J. Branovets, A. Hallikma, P. Nigumann, and M. Speek. 2011. Intronic L1 retrotransposons and nested genes cause transcriptional interference by inducing intron retention, exonization and cryptic polyadenylation. PLoS One 6:e26099.

6. Guo, W., S. Liu, Y. Cheng, L. Lu, J. Shi, G. Xu, N. Li, K. Cheng, et al. 2015. ICAM-1-related non-coding RNA in cancer stem cells maintains ICAM-1 expression in hepatocellular carcinoma. Clin Cancer Res. 22:2041-2050.

7. Wei, N., Y. Wang, R.X. Xu, G.Q. Wang, Y. Xiong, T.Y. Yu, G.S. Yang, and W.J. Pang. 2015. PU.1 antisense IncRNA against its mRNA translation promotes adipogenesis in porcine preadipocytes. Anim. Genet. 46:133-140.

8. Li, K., Y. Blum, A. Verma, Z. Liu, K. Pramanik, N.R. Leigh, C.Z. Chun, G.V. Samant, et al. 2010. A noncoding antisense RNA in tie-1 locus regulates tie-1 function in vivo. Blood 115:133-139.

9. Mahmoudi, S., S. Henriksson, M. Corcoran, C. Mendez-Vidal, K.G. Wiman, and M. Farnebo. 2009. Wrap53, a natural p53 antisense transcript required for p53 induction upon DNA damage. Mol. Cell 33:462-471.

10. Annilo, T., K. Kepp, and M. Laan. 2009. Natural antisense transcript of natriuretic peptide precursor A (NPPA): structural organization and modulation of NPPA expression. BMC Mol. Biol. 10:81.
11. Ogawa, Y., B.K. Sun, and J.T. Lee. 2008. Intersection of the RNA interference and $\mathrm{X}$-inactivation pathways. Science 320:1336-1341.

12. Rossi, G., M.V. Magni, and E. Albi. 2007. Sphingomyelin-cholesterol and double stranded RNA relationship in the intranuclear complex. Arch. Biochem. Biophys. 459:27-32.

13. Pruunsild, P., A. Kazantseva, T. Aid, K. Palm, and T. Timmusk. 2007. Dissecting the human BDNF locus: bidirectional transcription, complex splicing, and multiple promoters. Genomics 90:397-406.

14. Yin, G.L., Q. Chen, and W.J. Yang. 2007. Naturally occurring antisense RNA of allatostatin gene in the prawn, Macrobrachium rosenbergii. Comp. Biochem. Physiol. B Biochem. Mol. Biol. 146:20-25.

15. Bartsch, H., S. Voigtsberger, G. Baumann, I. Morano, and H.P. Luther. 2004. Detection of a novel sense-antisense RNA-hybrid structure by RACE experiments on endogenous troponin I antisense RNA. RNA 10:1215-1224.

16. Nishimura, Y., E.A. Kikis, S.L. Zimmer, Y. Komine, and D.B. Stern. 2004. Antisense transcript and RNA processing alterations suppress instability of polyadenylated mRNA in chlamydomonas chloroplasts. Plant Cell 16:2849-2869.

17. Okano, H., J. Aruga, T. Nakagawa, C. Shiota, and K. Mikoshiba. 1991. Myelin basic protein gene and the function of antisense RNA in its repression in myelin-deficient mutant mouse. J. Neurochem. 56:560-567.

18. Dreyfuss, G., V.N. Kim, and N. Kataoka. 2002. Messenger-RNA-binding proteins and the messages they carry. Nat. Rev. Mol. Cell Biol. 3:195205.

19. Speek, M. and W.L. Miller. 1995. Hybridization of the complementary mRNAs for P450c21 (steroid 21 -hydroxylase) and tenascin- $X$ is prevented by sequence-specific binding of nuclear proteins. Mol. Endocrinol. 9:1655-1665.

20. Krystal, G.W., B.C. Armstrong, and J.F. Battey. 1990. N-myc mRNA forms an RNA-RNA duplex with endogenous antisense transcripts. Mol. Cell. Biol. 10:4180-4191.

21. Schönborn, J., J. Oberstrass, E. Breyel, J. Tittgen, J. Schumacher, and N. Lukacs. 1991 Monoclonal antibodies to double-stranded RNA as probes of RNA structure in crude nucleic acid extracts. Nucleic Acids Res. 19:2993-3000.

Triin Mölder and Mart Speek

Department of Gene Technology, Tallinn University of Technology, Tallinn, Estonia

BioTechniques 61:61-65 (August 2016)

doi 10.2144/000114441

Keywords: guanidinium thiocyanate, RNA-RNA hybridization, RNA isolation, TRIzol, hnRNP

Supplementary material for this article is available at www.BioTechniques.com/article/114441.

Received 17 February 2016; accepted 09 May 2016

Address correspondence to Mart Speek, Department of Gene Technology, Akadeemia tee 15, Room 129 , Tallinn University of Technology, Tallinn 19086, Estonia. E-mail: mart.speek@ttu.ee.

To purchase reprints of this article, contact: biotechniques@fosterprinting.com

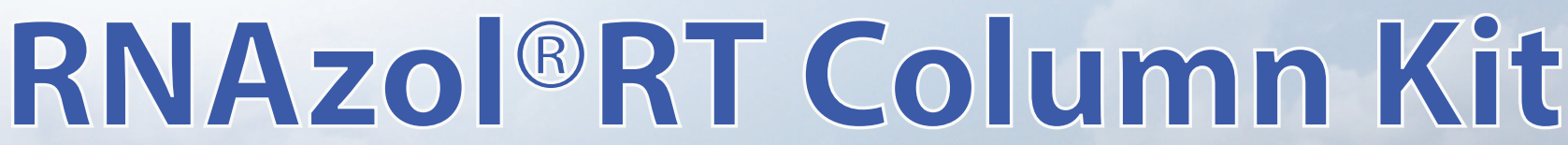

Universal RNA isolation kit from Molecular Research Center The market leader providing the best RNA isolation methods for over 25 years

Fast and dependable isolation

Highest yield and purity

No DNase treatment necessary
Process small or large samples Up to $\mathbf{3 0 0}$ mg RNA per column Variety of solid or liquid samples

\section{Specialized protocols for the isolation of:}

Total RNA containing all cellular RNA Large RNA containing mRNA, rRNA and non-coding RNA Small RNA containing micro RNA total RNA

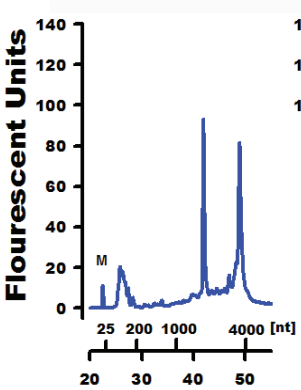

large RNA

small RNA
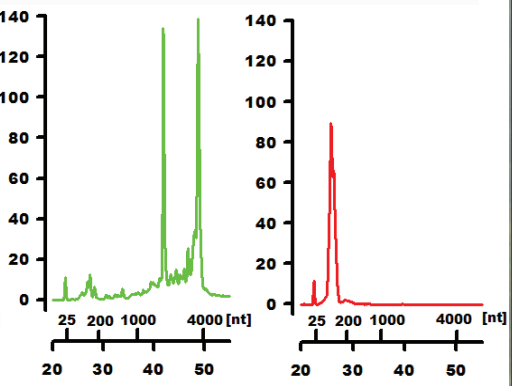

Time (sec) 